\title{
REVERBERATION MAPPING OF HIGH-LUMINOSITY QUASARS: FIRST RESULTS
}

\author{
Shai Kaspi, ${ }^{1,2}$ W. N. Brandt, ${ }^{3}$ Dan Maoz, ${ }^{1}$ Hagai Netzer, ${ }^{1}$ Donald P. Schneider, ${ }^{3}$ and Ohad Shemmer ${ }^{3}$ \\ Received 2006 September 7; accepted 2006 December 19
}

\begin{abstract}
Reverberation mapping of nearby active galactic nuclei (AGNs) has led to estimates of broad-line region (BLR) sizes and central object masses for some 37 objects to date. However, successful reverberation mapping has yet to be performed for quasars of either high luminosity (above $L_{\text {opt }} \sim 10^{46} \mathrm{ergs} \mathrm{s}^{-1}$ ) or high redshift $(z \gtrsim 0.3)$. Over the past 6 years, we have carried out, at the Hobby-Eberly Telescope, rest-frame ultraviolet spectrophotometric monitoring of a sample of six quasars at redshifts $z=2.2-3.2$, with luminosities of $L_{\mathrm{opt}} \sim 10^{46.4}-10^{47.6} \mathrm{ergs} \mathrm{s}^{-1}$, an order of magnitude greater than those of previously mapped quasars. The six quasars, together with an additional five having similar redshift and luminosity properties, were monitored photometrically at the Wise Observatory during the past decade. All 11 quasars monitored show significant continuum variations of order $10 \%-70 \%$. This is about a factor of 2 smaller variability than for lower luminosity quasars monitored over the same rest-frame period. In the six objects that have been spectrophotometrically monitored, significant variability is detected in the C IV $\lambda 1550$ broad emission line. In several cases the variations track the continuum variations in the same quasar, with amplitudes comparable to, or even greater than, those of the corresponding continua. In contrast, no significant Ly $\alpha$ variability is detected in any of the four objects in which it was observed. Thus, UV lines may have different variability trends in high-luminosity and low-luminosity AGNs. For one quasar, S5 0836+71 at $z=2.172$, we measure a tentative delay of 595 days between $\mathrm{C}$ IV and UV continuum variations, corresponding to a rest-frame delay of 188 days and a central black hole mass of $2.6 \times 10^{9} M_{\odot}$.

Subject headings: galaxies: active — galaxies: nuclei — galaxies: Seyfert — quasars: general

Online material: color figure
\end{abstract}

\section{INTRODUCTION}

Reverberation mapping has been used in the past two decades to estimate the sizes of the broad-line emitting regions (BLRs) in several dozens of active galactic nuclei (AGNs), and thus to infer the masses of the black holes at their centers (e.g., Kaspi et al. 2000). The technique uses the light-travel time-delayed flux response of the BLR to changes in the continuum flux (for reviews, see Peterson 1993, 2006; Netzer \& Peterson 1997, and references therein). Recently, a compilation of all available reverberation mapping data, for 37 AGNs, was analyzed in a uniform and selfconsistent manner. The relationship between luminosity, $L$, and the BLR size, $R_{\mathrm{BLR}}$, in several luminosity bands was studied by Kaspi et al. (2005), and the black hole mass-luminosity relation, $M_{\mathrm{BH}}-L$, based on these data was rederived by Peterson et al. (2004). Current reverberation studies cover 4 orders of magnitude in AGN luminosity in a well-sampled manner, from $\lambda L_{\lambda}(5100 \AA) \sim 10^{42}$ to $10^{46} \mathrm{ergs} \mathrm{s}^{-1}$. In this range, $R_{\mathrm{BLR}} \propto L^{\alpha}$, with $\alpha=0.67 \pm 0.05$ for the optical continuum, confirming the relations found by Kaspi et al. (2000; cf. Bentz et al. 2006, who found $\alpha=0.518 \pm 0.039$ ) and $M_{\mathrm{BH}} \propto L^{\beta}$, with $\beta=0.79 \pm 0.09$. In a recent study Peterson et al. (2005) measured the BLR size of the C IV $\lambda 1550$ emission line in the least-luminous AGN, NGC 4395, which has an optical luminosity of $\lambda_{\lambda}(5100 \AA)=5.9 \times 10^{39} \mathrm{ergs} \mathrm{s}^{-1}$. They found the $\mathrm{C}$ IV BLR size to be $1 \pm 0.3 \mathrm{lt}-\mathrm{hr}$, consistent with the size expected from extrapolating the $R_{\mathrm{BLR}}-L$ relation to lower luminosities.

The relations between AGN luminosity, BLR size, and black hole mass have been widely used for "single-epoch" estimates

\footnotetext{
1 School of Physics and Astronomy, Raymond and Beverly Sackler Faculty of Exact Sciences, Tel Aviv University, Tel Aviv 69978, Israel; shai@wise.tau ac.il.

${ }^{2}$ Physics Department, Technion, Haifa 32000, Israel.

3 Department of Astronomy and Astrophysics, Pennsylvania State University, University Park, PA 16802.
}

of black hole masses and accretion rates in studies addressing the issues of AGN accretion history and black hole growth (e.g., McLure \& Dunlop 2004; Barger et al. 2005; Yu et al. 2005; Kollmeier et al. 2006). However, these studies necessarily extrapolate the relations to luminosities and redshifts well beyond the ranges in which they were measured (e.g., McLure \& Jarvis 2002; Vestergaard 2002; Woo \& Urry 2002; Vestergaard \& Peterson 2006), since the only measured relations are in the luminosity range $10^{42}-10^{46} \mathrm{ergs} \mathrm{s}^{-1}$ and for redshift $<0.3$. As a result, the conclusions of such studies depend on the untested assumption that these extrapolations are valid. Although a posteriori explanations of the physical plausibility of the observed relations can be found, it is quite possible that subtle or strong deviations from the relations occur at high luminosities or redshifts (Netzer 2003). It is well known that some other AGN properties, such as the optical-to-X-ray spectral slope and emission-line equivalent widths (EWs), depend on luminosity (e.g., Baldwin 1977; Strateva et al. 2005; Steffen et al. 2006). AGN outflows also seem to be different between Seyfert galaxies (several hundred kilometers per second) and broad absorption line (BAL) quasars (several thousand to tens of thousands of kilometers per second), and thus might depend on luminosity (Laor \& Brandt 2002). Actual reverberation measurements for AGN with high luminosities and redshifts are therefore desirable.

However, reverberation mapping of high-luminosity quasars is an ambitious undertaking. Quasars of the highest luminosities (with bolometric luminosity $L_{\text {bol }} \approx 10^{47}-10^{48} \mathrm{ergs} \mathrm{s}^{-1}$ ) are expected to harbor some of the most massive black holes known, with $M_{\mathrm{BH}} \gtrsim 10^{9} M_{\odot}$. More massive black holes may have slower continuum flux variations with smaller amplitudes. This has been observed in X-rays (e.g., Lawrence \& Papadakis 1993; Uttley et al. 2002; Markowitz et al. 2003; O'Neill et al. 2005), and possibly in the UV-optical bands (e.g., Giveon et al. 1999; Cid Fernandes et al. 2000; Vanden Berk et al. 2004). The required 
TABLE 1

OBJect Characteristics

\begin{tabular}{|c|c|c|c|c|c|c|c|c|c|c|c|}
\hline $\begin{array}{l}\text { Object } \\
\text { (1) }\end{array}$ & $\begin{array}{l}\text { R.A. } \\
\text { (J2000.0) } \\
(2)\end{array}$ & $\begin{array}{c}\text { Decl. } \\
(\mathrm{J} 2000.0) \\
(3)\end{array}$ & $\begin{array}{l}m_{V} \\
(4)\end{array}$ & $\begin{array}{l}\text { Redshift } \\
\text { (5) }\end{array}$ & $\begin{array}{c}N_{\text {phot }} \\
\text { (6) }\end{array}$ & $\begin{array}{c}N_{\text {spec }} \\
\text { (7) }\end{array}$ & $\begin{array}{c}\lambda L_{\lambda}(5100 \AA) \\
(8)\end{array}$ & $\begin{array}{l}R_{\text {comp }} \\
\quad(9)\end{array}$ & $\begin{array}{l}\text { P.A.comp } \\
\text { (10) }\end{array}$ & $\begin{array}{c}R \\
(11)\end{array}$ & $\begin{array}{l}\text { Exp. } \\
(12)\end{array}$ \\
\hline \multicolumn{12}{|c|}{ Photometric and Spectrophotometric } \\
\hline S4 $0636+68 \ldots \ldots \ldots \ldots \ldots \ldots$ & 64204.2 & 675836 & 16.6 & 3.180 & 90 & 11 & 47.28 & 131.9 & 36.6 & 133.2 & 900 \\
\hline 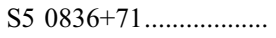 & 84124.3 & 705342 & 16.5 & 2.172 & 70 & 16 & 46.81 & 66.7 & 341.2 & 10064.5 & 600 \\
\hline SBS $1116+603 \ldots \ldots \ldots \ldots . . .$. & 111914.3 & 600457 & 17.5 & 2.646 & 85 & 15 & 46.92 & 147.2 & 62.7 & 632.7 & 900 \\
\hline SBS $1233+594 \ldots \ldots \ldots \ldots$ & 123549.5 & 591027 & 16.5 & 2.824 & 76 & 15 & 46.97 & 125.6 & 79.0 & 1.3 & 600 \\
\hline SBS $1425+606 \ldots \ldots \ldots \ldots . . . .$. & 142656.2 & 602551 & 16.5 & 3.192 & 90 & 21 & 47.43 & 78.6 & 2.4 & 4.6 & 300 \\
\hline HS $1700+6416 \ldots \ldots \ldots \ldots . . .$. & 170100.6 & 641209 & 16.1 & 2.736 & 96 & 17 & 47.49 & 91.4 & 135.4 & 3.6 & 300 \\
\hline \multicolumn{12}{|c|}{ Photometric } \\
\hline 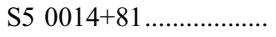 & 01708.5 & 813508 & 16.5 & 3.366 & 82 & $\ldots$ & 47.56 & $\ldots$ & $\ldots$ & 493.3 & $\ldots$ \\
\hline S5 $0153+74 \ldots \ldots$ & 15734.9 & 744243 & 16.0 & 2.338 & 65 & $\ldots$ & 46.85 & $\ldots$ & $\ldots$ & 12377.8 & $\ldots$ \\
\hline TB $0933+733 \ldots \ldots \ldots \ldots . . . .$. & 93748.8 & 730158 & 17.3 & 2.528 & 86 & $\ldots$ & 47.07 & $\ldots$ & $\ldots$ & 5.2 & $\ldots$ \\
\hline HS $1946+7658 \ldots \ldots \ldots \ldots . . . .$. & 194455.0 & 770552 & 15.8 & 2.994 & 102 & $\ldots$ & 47.63 & $\ldots$ & $\ldots$ & 1.7 & $\ldots$ \\
\hline S5 $2017+74 \ldots \ldots \ldots \ldots \ldots \ldots$ & 201713.1 & 744048 & 18.1 & 2.187 & 77 & $\ldots$ & 46.44 & $\ldots$ & $\ldots$ & 2765.8 & $\ldots$ \\
\hline
\end{tabular}

Notes._- Col. (1): Object name. Cols. (2)-(3): Right ascension and declination from NED. Units of right ascension are hours, minutes, and seconds, and units of

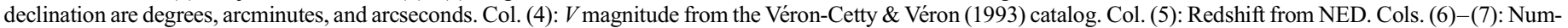

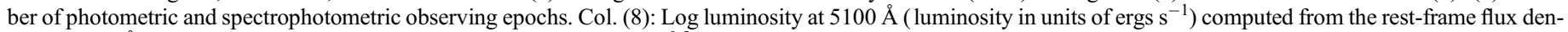

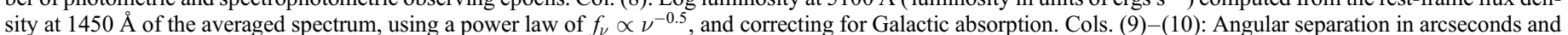

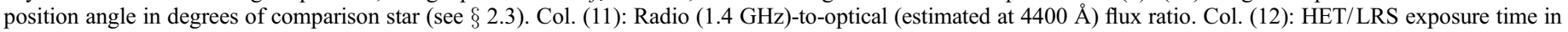
seconds (two consecutive exposures with this exposure time were obtained each visit).

observing periods of high-luminosity quasars are also significantly lengthened by cosmological time dilation, since such sources are typically found at high redshifts $(z \gtrsim 1)$. On the other hand, the ability to monitor high- $z$ objects in the rest-frame UV, in which AGN variability amplitudes are routinely higher than in the optical, can lead to better characterized continuum light curves. The smaller intrinsic variability amplitude of the continuum could result in smaller flux-variability amplitudes for the emission lines, affecting the ability to detect the time delay in the BLR response. Furthermore, since the EWs of high-ionization emission lines decrease with increasing luminosity (the well-known "Baldwin effect"; Baldwin 1977), fluxes of emission lines such as C iv $\lambda 1550$, which are most suitable for such studies, are harder to measure. On the other hand, the fluxes in the UV lines are generally higher than those of the Balmer lines used in rest-frame optical studies. Finally, high-redshift sources are fainter and hence more difficult to observe. Probably due to all of these possible problems, no reverberation measurements exist for AGNs with $L \gtrsim 10^{46} \mathrm{ergs} \mathrm{s}^{-1}$, and several attempts at such measurements have so far not been successful (e.g., Welsh et al. 2000; Trevese et al. 2006; A. Marconi 2005 , private communication).

In view of the many unknowns and the opposing effects entering the above discussion, and considering the importance of the subject, over a decade ago, we began a reverberation mapping program aimed at high-luminosity, high-redshift AGNs. In this paper, we describe our program and present some initial results. In $\S 2$ we describe the sample, the observations, and the data reduction. In $\S 3$ we perform a time series analysis for the AGN light curves and discuss our results, with a summary in $\S 4$. Throughout this paper we use the standard cosmology with $H_{0}=70 \mathrm{~km} \mathrm{~s}^{-1} \mathrm{Mpc}^{-1}$, $\Omega_{M}=0.3$, and $\Omega_{\Lambda}=0.7$ (Spergel et al. 2003; Riess et al. 2004).

\section{SAMPLE, OBSERVATIONS, AND DATA REDUCTION}

\subsection{Sample Selection}

We selected our sample of 11 high-luminosity quasars in 1994 from the Véron-Cetty \& Véron (1993) catalog. The selection cri- teria were observationally oriented: a high declination $\left(\delta \gtrsim 60^{\circ}\right)$, so as to make the quasars observable from the Northern Hemisphere for most of the year; observed magnitude $V \lesssim 18$, to permit the acquisition of low-resolution, high signal-to-noise $(\mathrm{S} / \mathrm{N})$ spectra within a reasonable observing time; and, since high-luminosity quasars are known only at high redshifts, redshifts in the range $2<z<3.4$ that bring the prominent broad UV emission lines ( $\mathrm{C}$ IV and $\operatorname{Ly} \alpha$ ) into the optical range. We chose the 11 objects with the brightest apparent optical magnitudes that satisfy these criteria. The sample is listed in Table 1 . The choice of bright quasars at high redshift naturally favors quasars with high luminosity. The luminosity range of our sample is $10^{46.4} \lesssim \lambda L_{\lambda}(5100 \AA) \lesssim$ $10^{47.6} \mathrm{ergs} \mathrm{s}^{-1}$. This is an order of magnitude higher than other AGNs with existing reverberation measurements.

In Figure 1 we plot the absolute $i$ magnitude versus redshift for all objects in the Sloan Digital Sky Survey (SDSS; York et al. 2000) Data Release 3 (DR3; Abazajian et al. 2005) Quasar Catalog (Schneider et al. 2005), compared to our sample and to other objects with reverberation mapping data. Since the SDSS DR3 quasar catalog covers about $10 \%$ of the sky, future surveys will not find large numbers of quasars significantly more luminous than those in it, and thus the objects in our sample are clearly among the most luminous quasars in the universe. Our sample includes HS $1700+6416$ and SBS $1425+606$, which are the two most luminous quasars in DR3. Our study is probing powerful quasars at redshifts near the peak of their comoving number density (near $z \sim 2$ ), which is the main era of black hole growth for the most massive black holes and galaxies. Figure 1 also shows that there are only two objects with $M_{i}<-26$ that have a previously measured BLR size, which illustrates the gap in luminosity between our sample and previously studied AGNs.

The luminosities of the quasars in our sample are likely intrinsic, rather than being affected by gravitational lensing magnification. To our knowledge, none of the objects is a lensed quasar. In particular, five of the quasars were imaged with the Hubble Space Telescope by Maoz et al. (1993), who found no evidence for strong lensing. Maoz et al. (1993) and other optical lensed 


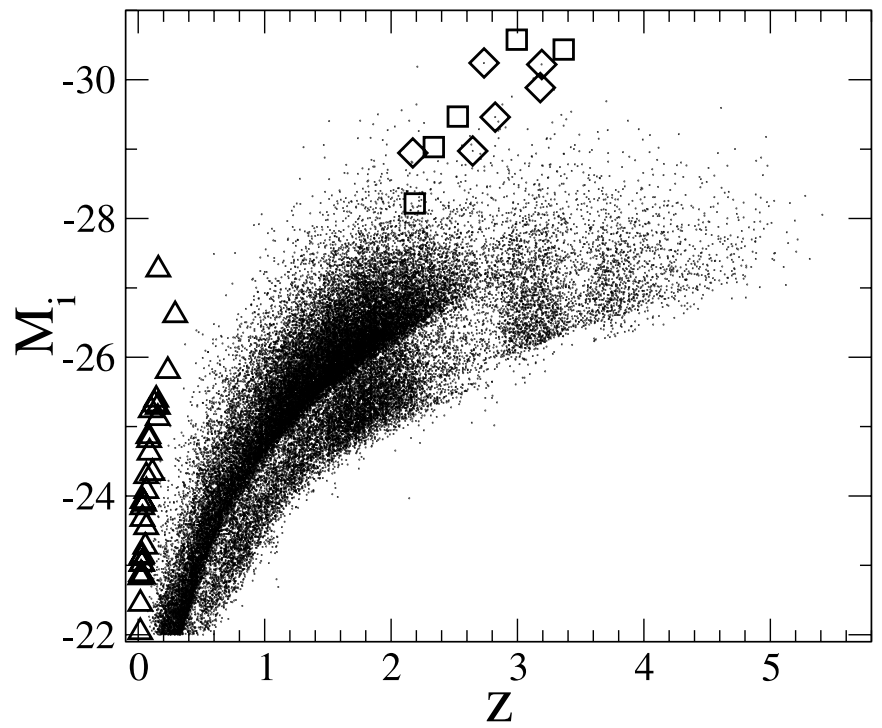

FIG. 1.- Absolute $i$ magnitude vs. redshift for all SDSS DR3 quasars (dots). The six quasars monitored at the HET are marked with diamonds, and the five objects from our sample with photometric data only are marked with squares. AGNs with reverberation mapping data are marked with triangles. The objects in our sample are clearly among the most luminous quasars and lie within the primary epoch of black hole growth for luminous quasars.

quasar surveys (e.g., Morgan et al. 2003) have consistently found a lensed fraction of $\sim 1 \%$ among luminous quasars, and thus it is not surprising that none among our 11 objects are lensed. We also note that none of our objects is a BAL quasar, and none have significant UV absorption lines.

Because the Véron-Cetty \& Véron (1993) catalog was a literature compilation, our sample is not statistically complete, nor necessarily representative of the luminous quasar population. For example, six of the quasars in our sample (i.e., about 55\%) are radio loud, somewhat more than the $\sim 20 \%$ radio-loud fraction (i.e., two objects) one would expect for a quasar population of this mean redshift (2.8) and luminosity (Jiang et al. 2007). We note that the incompleteness of AGN reverberation samples applies to most of the studies that have derived BLR size-massluminosity relations. An exception is the sample of 17 PG quasars mapped by Kaspi et al. (2000), which were an unbiased selection from the full, flux-limited, color-selected Bright Quasar Survey (BQS; Schmidt \& Green 1983; also see Maoz et al. 1994). Of course, the PG sample itself suffers from some incompleteness; see Jester et al. (2005) and references therein. Although there are no obvious biases that are introduced into reverberation measurements by these samples (e.g., radio-loud and radio-quiet objects seem to follow the same BLR size-luminosity relation; see the few radio-loud objects in the Kaspi et al. [2000] sample and also Wu et al. [2004]), it should be kept in mind that such biases may, nonetheless, exist (see, e.g., Netzer 2003).

The observations and reductions for the current project follow the same principles as those of similar projects carried out by our group (e.g., Maoz et al. 1994; Netzer et al. 1996; Giveon et al. 1999; Kaspi et al. 2000). We outline the main features in the following sections.

\subsection{Broadband Imaging}

Imaging data have been obtained, starting in 1995, at the Wise Observatory (WO; Kaspi et al. 1995) $1 \mathrm{~m}$ telescope in the JohnsonCousins $B$ and $R$ bands, using a $1024 \times 1024$ back-illuminated Tektronix CCD. The exposure times are $250 \mathrm{~s}$ in $R$ and $300 \mathrm{~s}$ in $B$. Since the targets have high declinations, they can be observed from the WO for about 10 months a year, with observations scheduled about once every month (based on the experience with the PG quasars [Kaspi et al. 2000], and the expectation that the variability timescales of high-luminosity quasars are longer, this sampling rate was deemed sufficient). The data have been reduced using IRAF ${ }^{4}$ procedures in the standard way. Broadband light curves for the quasars are produced by comparing their instrumental magnitudes to those of constant-flux stars in the field (see, e.g., Netzer et al. 1996 for details). The uncertainties on the photometric measurements include the fluctuations due to photon statistics and the scatter (of 0.03 mag or less) in the measurement of the constant-flux stars used. The typical total uncertainty is in the range of 0.01-0.05 mag depending on object brightness and the observing conditions. The photometric light curves provide a check on any continuum variability detected in the spectroscopic observations and improve the sampling of the continuum light curves. Furthermore, the photometric observations began several years before the spectroscopic observations. In the analysis, this measurement of the continuum behavior at early times gives an enlarged baseline for the cross-correlation between the line and continuum light curves.

\subsection{Spectrophotometry}

Spectrophotometric monitoring of six of the 11 quasars has been carried out since 1999 at the $9 \mathrm{~m}$ Hobby-Eberly Telescope (HET; Ramsey et al. 1998; L. W. Ramsey et al. 2007, in preparation). The other five quasars have declinations above the HET's high-declination limit $\left(71^{\circ} 41^{\prime}\right)$. The operation of the HET in a queue-scheduled mode is a key component of our observing program; it enables ideal spreading of the observations throughout the observing season for each object and ensures that observations will not be lost due to poor weather. We have used the Low Resolution Spectrograph (LRS; Hill et al. 1998) with a 600 line $\mathrm{mm}^{-1}$ grating, a $10^{\prime \prime}$ wide slit, and the GG385 order separation filter. The spectral range covered with this configuration is $4300-7300 \AA$ with a resolution of about $20 \AA$. Typical seeing in the HET data is in the range $1.5^{\prime \prime}-3^{\prime \prime}$. Each night the standard calibration images of bias, sky-flat fields, and internal lamp flats are obtained. Exposures of $\mathrm{Ne}$ and $\mathrm{Cd}$ lamps are used for wavelength calibration (up to mid-2002 a Hg-Cd-Zn lamp was used). The objects in our sample are accessible to the HET for about 6 months each year. During each such period, we strive to obtain three observations separated by $\sim 2.5$ months. For each quasar, the spectrograph's focal plane is rotated to an appropriate position angle so that a nearby comparison star (cols. [9] and [10] in Table 1) is included simultaneously in the slit. This permits spectrophotometric calibration of the quasars under nonphotometric conditions. The wide slit reduces the effects of atmospheric dispersion at the nonparallactic position angles, as well as light losses due to guiding errors and poor seeing.

Observations typically consist of two consecutive exposures of the quasar/star pair. Exposure times range from 300 to $900 \mathrm{~s}$ for each exposure depending on object brightness, aiming for a $\mathrm{S} / \mathrm{N}$ of $>20$ pixel $^{-1}$ in the continuum. The spectroscopic data are reduced using standard IRAF routines. The images are bias and flat field corrected. The extraction width is typically $9.4^{\prime \prime}$, and wavelength calibration is applied to the data after the spectral extraction. This procedure results in two spectra from each image: one for the quasar and one for the comparison star. The

\footnotetext{
4 The Image Reduction and Analysis Facility (IRAF) is distributed by the National Optical Astronomy Observatory, which is operated by AURA, Inc., under cooperative agreement with the National Science Foundation.
} 
consecutive quasar/star flux ratios are then compared to test for systematic errors in the observations and to identify and remove cosmic-ray events. The ratio is usually reproducible to $0.5 \%-4 \%$ at all wavelengths; observations with ratios larger than $5 \%$ are discarded (this occurred in about $7 \%$ of the observations). We note a trend of improvement in the HET observations in image quality and seeing over the years due to improvement in its equipment and manner of operation. We have verified that all our comparison stars are nonvariable to within $\sim 2 \%$ by means of differential photometry with other stars in each field (see $\S 2.2)$.

Spectra were calibrated to an absolute flux scale using observations of spectrophotometric standard stars observed at several epochs. The uncertainty in the calibration is $\sim 20 \%$, which is not shown in the error bars in our light curves. The error bars reflect only the measurement and differential uncertainties, which are of order $1 \%-4 \%$. These are comparable to other reverberation mapping studies that have used the same observing technique (e.g., Kaspi et al. 1996b; Collier et al. 1998; Kaspi et al. 2000).

For each quasar, we used all available spectra to produce an average spectrum and an rms spectrum, defined as

$$
\sigma(\lambda)=\left\{\frac{1}{(N-1)} \sum_{i=1}^{N}\left[f_{i}(\lambda)-\bar{f}(\lambda)\right]^{2}\right\}^{1 / 2},
$$

where the sum is taken over the $N$ spectra, and $\bar{f}(\lambda)$ is the average spectrum (Peterson et al. 1998a). The average spectra of the six quasars in our sample are shown in Figure 2. We used the average and rms spectra to choose line-free spectral bands suitable for setting the continuum underlying the emission lines, and the wavelength limits for integrating the line fluxes. The line and continuum fluxes were measured algorithmically for all epochs by calculating the mean flux in the continuum bands, and summing the flux above a straight line in $f_{\lambda}$ that connects the continuum bands straddling each emission line. This process measures the total emission in each line, i.e., the flux of the broad component of the line together with its narrow component. We note, however, that in the high-ionization lines we monitor in luminous quasars, the narrow-line contribution is negligible (e.g., Wills et al. 1993). In any event, the narrow component is nonvariable on the timescales probed here and would merely contribute a constant flux level to the light curves.

The uncertainty in the line flux was estimated by propagating the uncertainty in the definition of the continuum levels, determined from the standard deviation of the mean in the continuum bands (of order $1 \%-3 \%$ ). To this we added in quadrature our estimate for the uncertainty in the calibration of the differential spectrophotometry, which is of order $1 \%-4 \%$.

\subsection{Continuum and Emission-Line Light Curves}

As seen in all the quasar spectra in Figure 2, the $R$ band $(\lambda \sim 6500 \AA)$ is dominated by continuum emission, with negligible contribution from broad emission lines. In contrast, emission lines contribute of order one-half the flux in the $B$ band for several of the quasars. The variations in the $R$-band photometry are therefore determined almost completely by continuum variations, and this broadband light curve can be merged with the spectroscopic continuum light curve to improve the sampling and duration of the continuum light curve. The photometric and spectrophotometric light curves of each object are intercalibrated by comparing all pairs of spectrophotometric and photometric observations

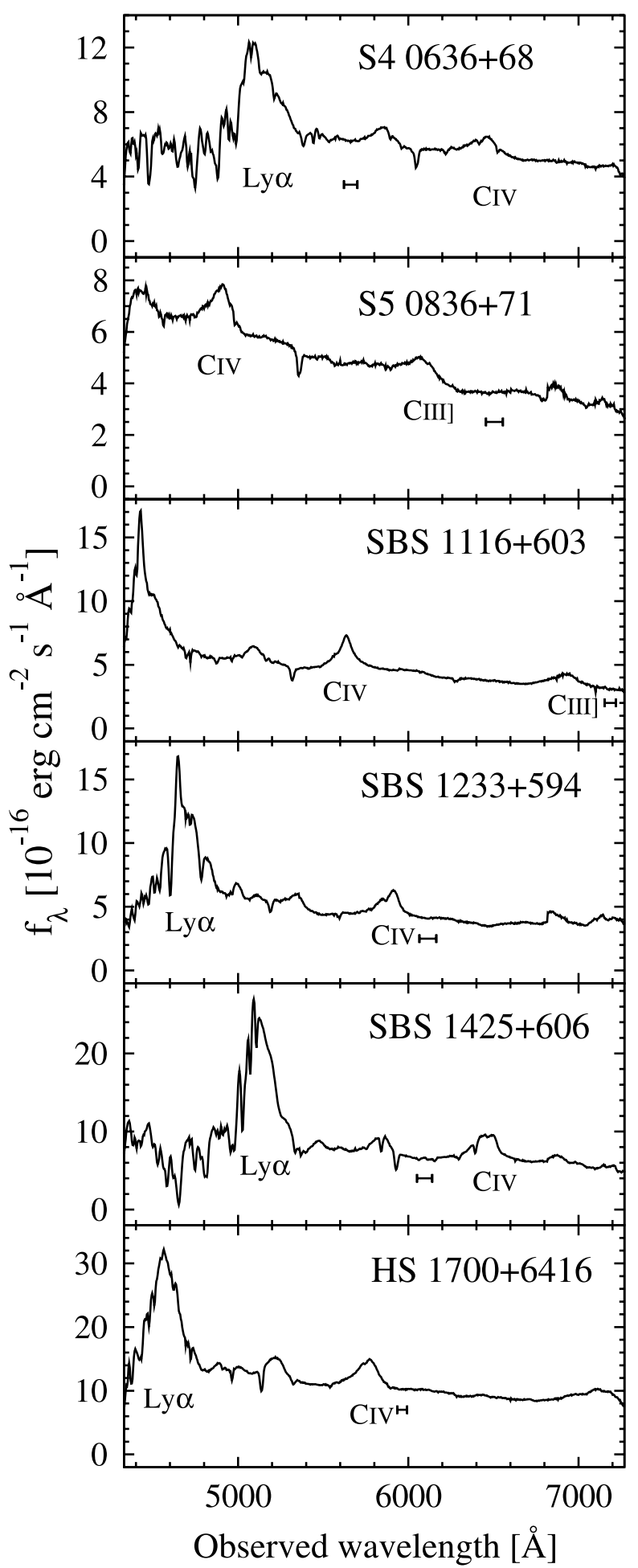

FIG. 2.-Mean spectra of the six quasars monitored with the HET. Each spectrum contains roughly a dozen individual observations. The spectral resolution is $\approx 20 \AA$. The line and continuum bands discussed in the text are marked in each panel.

separated in time by less than 20 days. For the data presented here, this produced $\sim 10$ pairs of points per object. A linear leastsquares fit between the spectrophotometric continuum fluxes and the photometric fluxes (the instrumental $R$ magnitudes were translated to fluxes) was then used to merge the two light curves. A clear relation was always apparent in this fitting, and a linear 
Month -Year

06-1995 06-1996 06-1997 06-1998 06-1999 06-2000 06-2001 06-2002 06-2003 06-2004

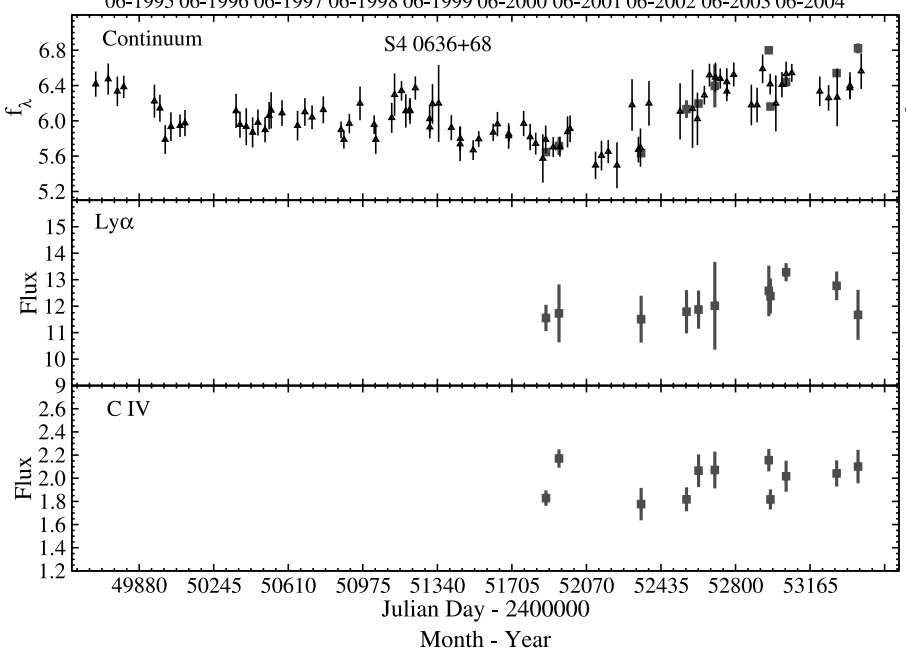

06-1995 06-1996 06-1997 06-1998 06-1999 06-2000 06-2001 06-2002 06-2003 06-2004

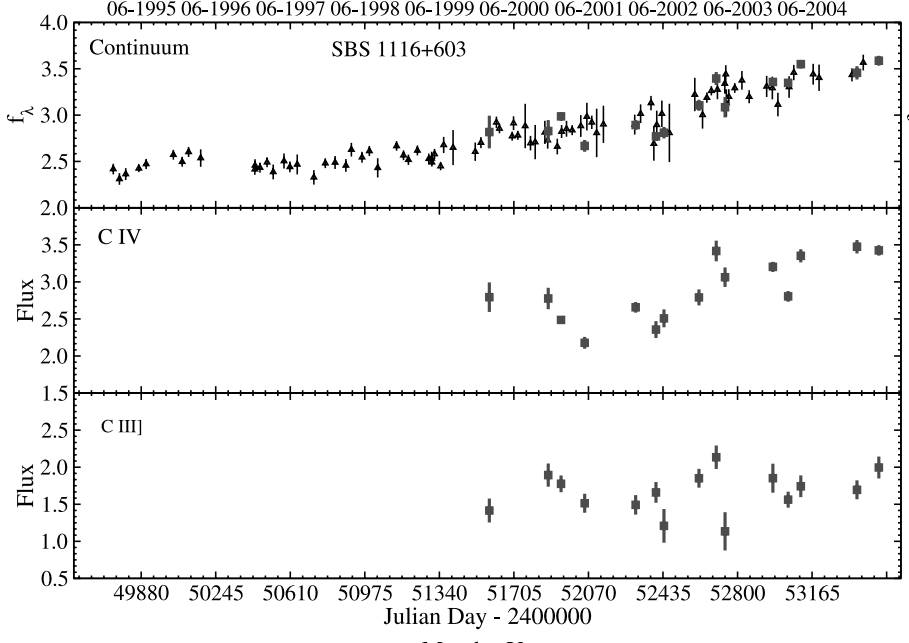

Month - Year

Month - Year
06-1995 06-1996 06-1997 06-1998 06-1999 06-2000 06-2001 06-2002 06-2003 06-2004

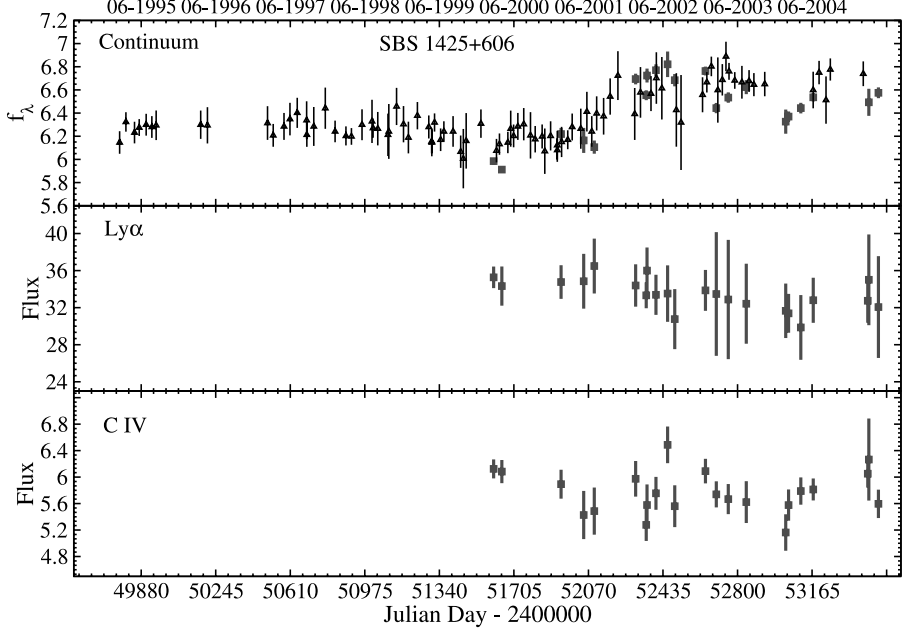

Month - Year

06-1995 06-1996 06-1997 06-1998 06-1999 06-2000 06-2001 06-2002 06-2003 06-2004

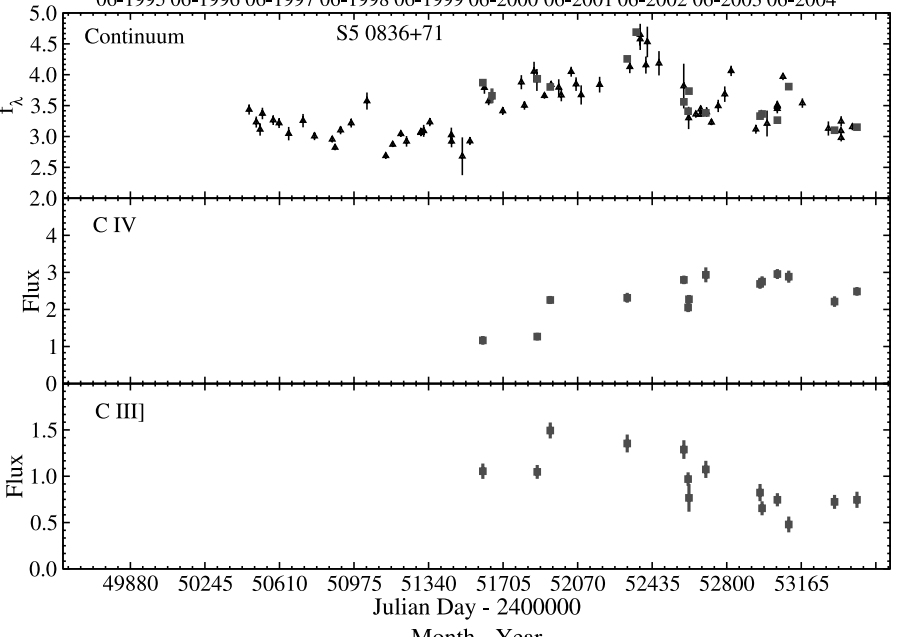

06-1995 06-1996 06-1997 06-1998 06-1999 06-2000 06-2001 06-2002 06-2003 06-2004

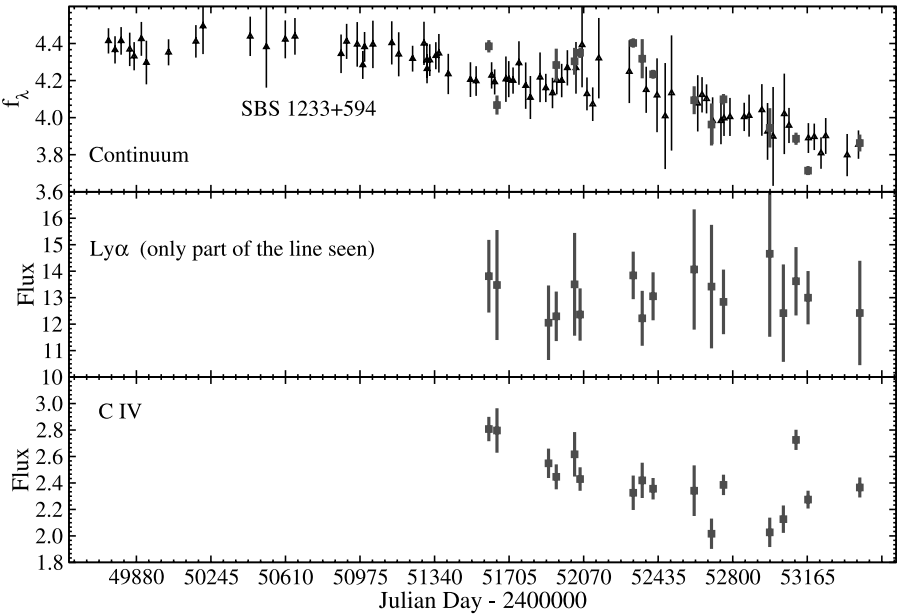

Month -Year

06-1995 06-1996 06-1997 06-1998 06-1999 06-2000 06-2001 06-2002 06-2003 06-2004

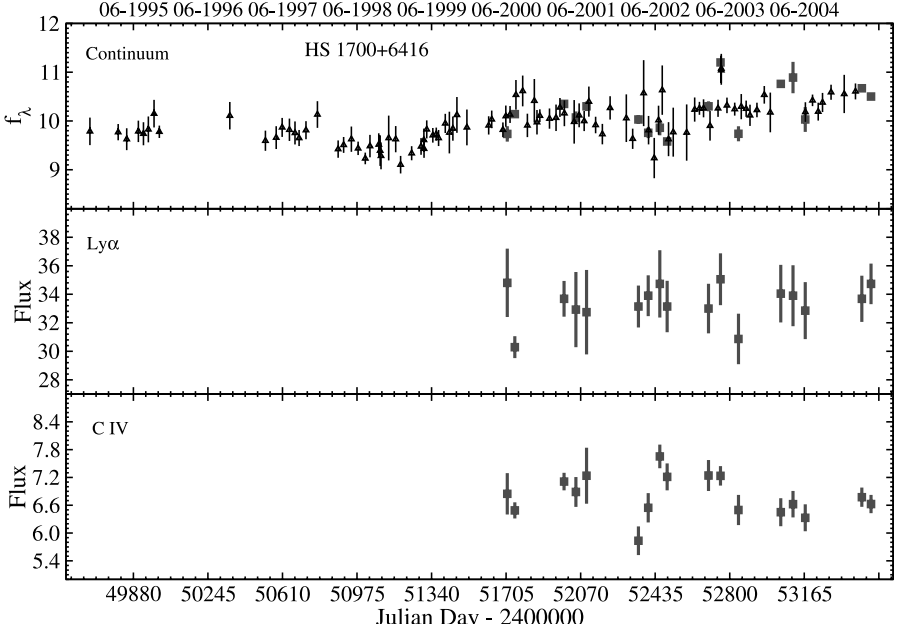

FIG. 3.- Light curves for the six quasars that have been monitored at the HET. Squares are spectrophotometric data from the HET. Triangles are photometric data from

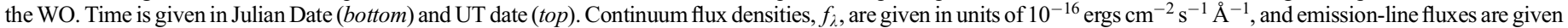
in units of $10^{-14} \mathrm{ergs} \mathrm{cm}^{-2} \mathrm{~s}^{-1}$. [See the electronic edition of the Journal for a color version of this figure.]

model was an adequate representation, indicating the continuum light curves from the spectroscopic and the photometric observations are in good agreement.

For each object, the total number of photometric and spectrophotometric observations is given in Table 1, columns (6) and (7), respectively (the sum of the two columns gives the total number of points in the continuum light curve). Note that for particular emission lines in several objects, some data points are missing because of insufficient wavelength coverage or low$\mathrm{S} / \mathrm{N}$. Light curves for the six quasars monitored at the HET are presented in Figure 3. The different bands used for the flux measurements in Figure 3 are listed in Table 2 for each object 
TABLE 2

InTEgRation Limits For Continuum Bands

\begin{tabular}{cc}
\hline Object & $\begin{array}{c}\text { Wavelength Range } \\
(\AA)\end{array}$ \\
\hline S4 $0636+68 \ldots \ldots \ldots \ldots \ldots \ldots .$. & $5622-5701$ \\
S5 $0836+71 \ldots \ldots \ldots \ldots \ldots \ldots$. & $6456-6556$ \\
SBS $1116+603 \ldots \ldots \ldots \ldots \ldots$. & $7154-7220$ \\
SBS $1233+594 \ldots \ldots \ldots \ldots \ldots$. & $6065-6165$ \\
SBS $1425+606 \ldots \ldots \ldots \ldots \ldots . .$. & $6051-6141$ \\
HS $1700+6416 \ldots \ldots \ldots \ldots \ldots$. & $5935-5993$ \\
\hline
\end{tabular}

(wavelengths are given in the observed frame); $R$-band light curves for the five objects monitored photometrically at the WO are presented in Figure 4.

\section{TIME SERIES ANALYSIS}

\subsection{Continuum and Line Variability}

In Table 3 we list variability measures for all light curves presented above. Column (1) lists the object name and column (2) the particular light curve. In columns (3), (4), and (5) we list the mean $(\bar{f})$, rms $(\sigma)$, and the mean uncertainty $(\delta)$ of all data points in the light curves, respectively, in the appropriate units for each light curve. In column (6) we list the $\chi_{\nu}^{2}$ obtained by fitting a constant to the light curve, and in column (7) we list $P\left(\chi^{2} \mid \nu\right)$, the probability to get such $\chi^{2}$ if there were intrinsically no variability. This test is used to determine whether the light curve is consistent with a constant flux to a significance level of $95 \%$. If the light curve passed the test, we list for it in column (8) the intrinsic normalized variability measure, $\sigma_{N}=100\left(\sigma^{2}-\delta^{2}\right)^{1 / 2} / \vec{f}$ (used by Kaspi et al. 2000), and in column (9) we list the fractional variability amplitude, $F_{\mathrm{var}}$, and its uncertainty (Rodriguez-Pascual et al. 1997; Edelson et al. 2002).

In general, all continuum light curves show variations of $10 \%-70 \%$ measured relative to the minimum flux. The mean intrinsic normalized variability measure (col. [8] of Table 3 ) is $5.8 \%$, and the median is $4.1 \%$. The points that appear to deviate strongly from the mean flux are generally due to unreliable measurements and have relatively large uncertainties. A possible exception is one particular deviation in the continuum light curve of HS 1700+6416 around JD 2,452,757 (2003 April 27), where the object seems to brighten (both in the spectroscopic and in the photometric measurements) by about $10 \%$ for $\sim 15$ days. This object was also monitored by Reimers et al. (2005), who found that HS $1700+6416$ was $20 \%$ brighter in the $R$ band on 2003 May $14-18$ than in 1995 March and 1998 July. This agrees with our measurements at the same epochs and suggests that the above deviation in the light curve is real.

It is instructive to compare the variability characteristics of our sample to those of the low-redshift PG quasar sample (Kaspi et al. 2000). However, one needs to consider the differences in the rest-frame monitoring periods of the two samples. While the continuum light curves of the high-luminosity quasars span over $10 \mathrm{yr}$ in the observed frame, this period corresponds to only about $2-3 \mathrm{yr}$ in their rest frames. We have therefore analyzed the first $3 \mathrm{yr}$ period in the $\mathrm{PG}$ quasar light curves (which span over $7.5 \mathrm{yr}$ ). The resulting variability measured for the PG quasar sample, $25 \%-125 \%$, is about twice the $10 \%-70 \%$ variability range of the high-luminosity quasars. Also, the mean and rms of $\sigma_{N}$ for the PG quasars (in the first $3 \mathrm{yr})$ is $\left\langle\sigma_{N}\right\rangle=(10.4 \pm 4.5) \%$ about twice that of the high-luminosity quasars, which have $\left\langle\sigma_{N}\right\rangle=$

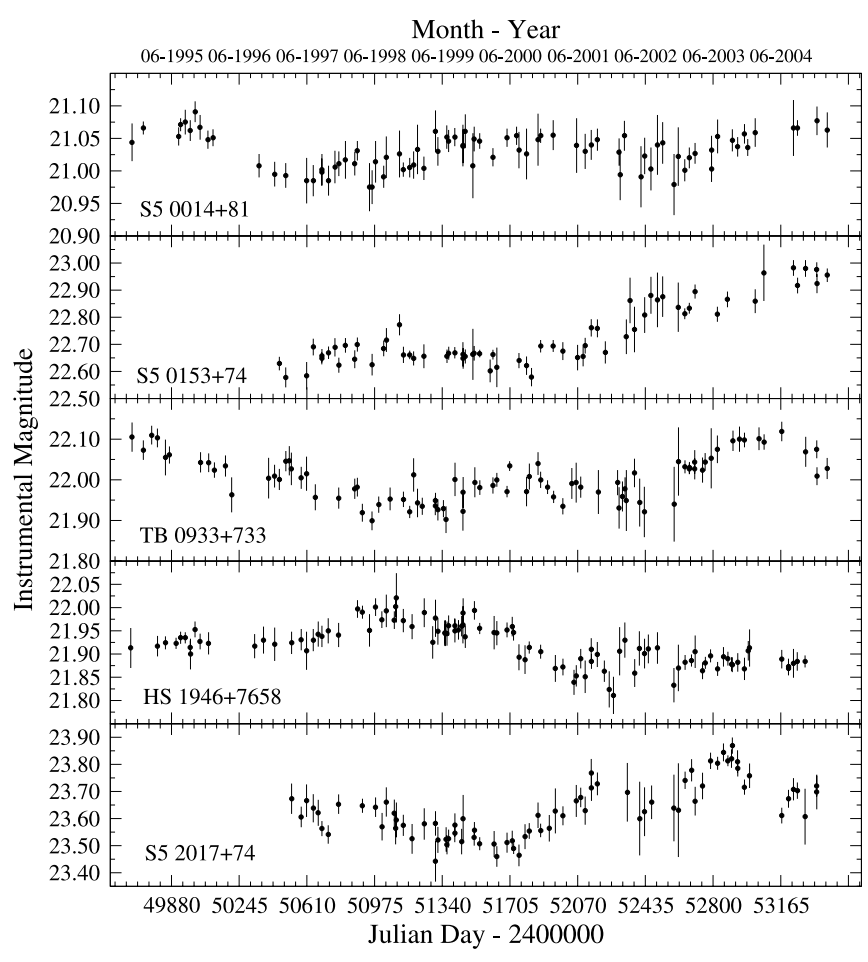

FIG. 4. - Plot of $R$-band light curves for the five quasars that have been monitored photometrically at the WO. Magnitudes are given in arbitrary units. Time is given in Julian Date (bottom) and UT date (top).

$(5.8 \pm 3.9) \%$. The lower rest-frame variability we measure in the continuum for the current sample is probably a manifestation of the general trend that high-luminosity AGNs have longer variability timescales (e.g., Vanden Berk et al. 2004), perhaps as a result of their higher black hole masses.

Proceeding to the emission-line light curves, the variability measures listed in Table 3 show that none of the four Ly $\alpha$ light curves show significant variability. In contrast, the two C III] $\lambda 1909^{5}$ light curves and all six $\mathrm{C}$ iv light curves show significant variability, with $F_{\text {var }}$ significant at the $\gtrsim 3 \sigma$ level. We note that the variability measures of these emission-line light curves are comparable to, or even greater than, those of their corresponding continuum light curves. There are few previous AGN UV data sets with which to compare these possible trends. The only quasar with UV variability data of similar quality is 3 C 273 . Interestingly, Ulrich et al. (1993) noted the nonvariability of Ly $\alpha$ in this object, at a level of $<5 \%$, over a period of $15 \mathrm{yr}$, despite factor of 2 variations in the continuum during the same period (there are no data for the $\mathrm{C}$ IV and $\mathrm{C}$ III] lines during that time). In contrast, Seyfert galaxies seem to show comparable variation amplitudes in the UV continuum and in the $\mathrm{Ly} \alpha, \mathrm{C}$ IV, and C III] lines, although the line variation amplitudes tend to be somewhat smaller than the continuum variations. This has been seen in NGC 5548 (Clavel et al. 1991; Korista et al. 1995), NGC 7469 (Wanders et al. 1997), NGC 3783 (Reichert et al. 1994), Fairall 9 (RodriguezPascual et al. 1997), and 3C 390.3 (O'Brien et al. 1998).

The indication that, in quasars, the $\mathrm{C}$ IV, $\mathrm{C} \mathrm{III]}$, and Ly $\alpha$ lines show different variability trends may be related to the fact that each line is driven by a different ionizing continuum. This could also explain the different variability amplitudes, e.g., if the Ly $\alpha$ line is driven by a weakly varying UV continuum, while the C IV

5 The $\mathrm{C}$ III] complex we measure includes up to $\sim 50 \%$ flux contribution from Si III] $\lambda 1892$ and Fe III multiplets. 
TABLE 3

Variability Measures

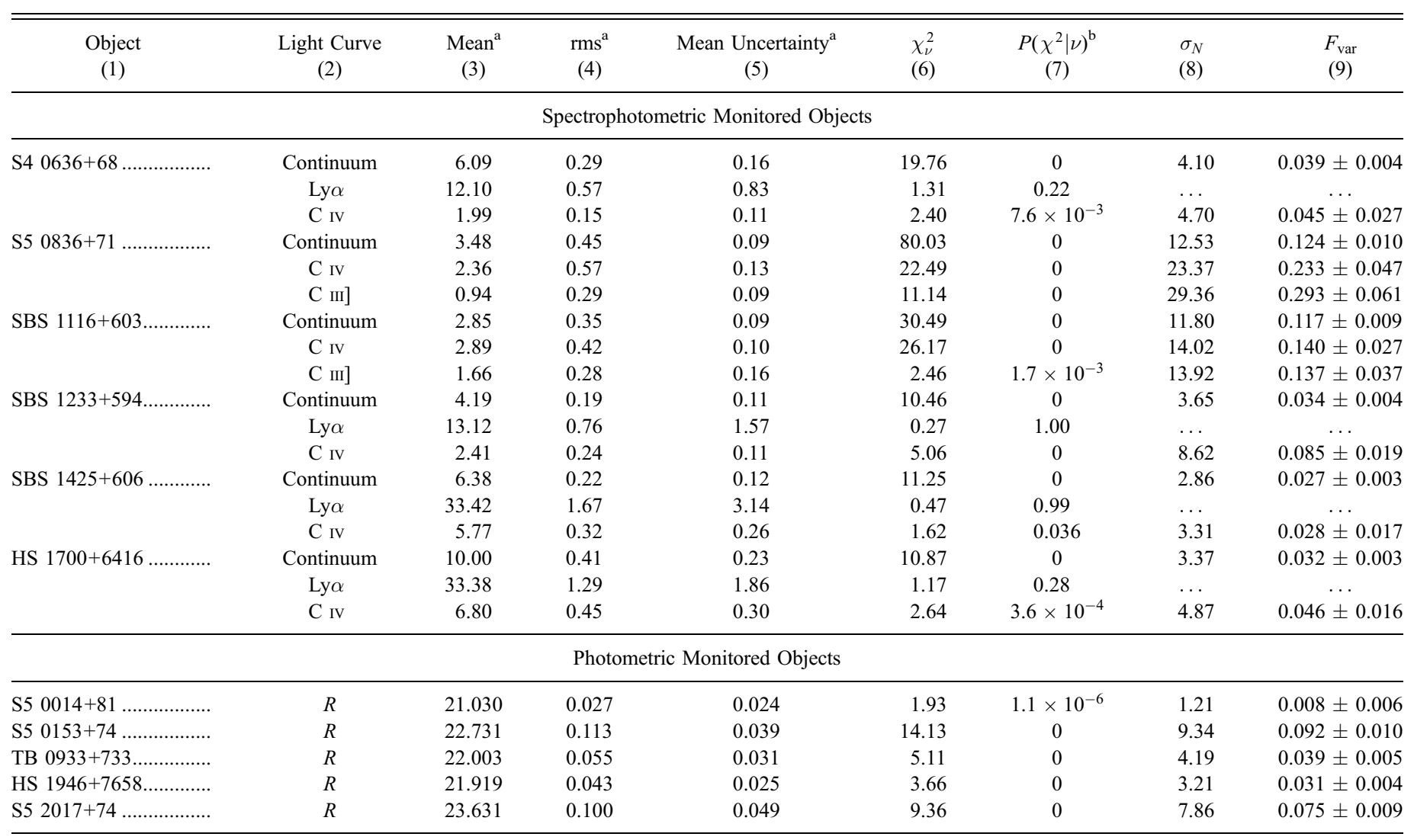

Notes.-No data in Cols. (8) and (9) means that no significant variability was detected in this light curve. In particular, none of the Ly $\alpha$ light curves show significant variability.

${ }^{a}$ The units are $10^{-16} \mathrm{ergs} \mathrm{cm}{ }^{-2} \mathrm{~s}^{-1} \AA^{-1}$ for the continuum light curves, $10^{-14} \mathrm{ergs}^{-2} \mathrm{~s}^{-1}$ for the emission-line light curves, and instrumental magnitude for the $R$-band light curves.

${ }^{\mathrm{b}}$ Probabilities smaller than $10^{-9}$ are listed as zero.

is controlled by a more strongly varying extreme-UV to X-ray continuum. Indeed, the continuum variability amplitudes of AGN are observed to grow with decreasing wavelength, from optical through the UV and to the X-rays, and the variations are sometimes poorly correlated with each other in different bands (e.g., Maoz et al. 2002; Shemmer et al. 2003). The differences in response behaviors among quasars and Seyferts would then imply that the degree to which continuum variations grow with decreasing wavelength depends on luminosity. However, the current data are still sparse, and the above trends require confirmation.

Comparison of the variability in the emission-line light curves between the PG sample and the present high-luminosity sample is complicated by the fact that, in the PG sample, the Balmer lines were monitored, while for the high-luminosity quasars we monitor UV lines. Furthermore, the high-luminosity quasar lines were monitored for only half the period over which the continuum was monitored, i.e., approximately $5 \mathrm{yr}$, which is about $1.2-1.5 \mathrm{yr}$ in the quasar rest frames. Analyzing only the first $1.5 \mathrm{yr}$ of the Balmer line light curves of the PG quasars, we find that about one-third of the lines did not vary; most of the nonvarying light curves are for the $\mathrm{H} \gamma$ line, which has lower EW than the $\mathrm{H} \alpha$ and $\mathrm{H} \beta$ lines. If, for the $\mathrm{PG}$ quasar emission lines, we average the variability measures of only $\mathrm{H} \alpha$ and $\mathrm{H} \beta$, we find $\left\langle\sigma_{N}\right\rangle=(7.5 \pm$ $6.2) \%$ (mean and rms), compared to the average variability measure of the $\mathrm{C}$ III] and $\mathrm{C}$ Iv lines of the high-luminosity quasars in the current sample, $\left\langle\sigma_{N}\right\rangle=(12.8 \pm 9.4) \%$. It therefore appears that the UV lines may be more responsive than the Balmer lines to continuum variations, which could compensate for the lower continuum variability amplitudes at high luminosities, and contribute to the success of our experiment. Indeed, the high ratio of emission-line to continuum variation amplitudes in the highluminosity sample is in contrast to the common behavior in the PG quasars, which show less variability in the Balmer lines than in the continuum. (Some low-luminosity AGNs, e.g., NGC 4051 [Shemmer et al. 2003], do show larger variability amplitudes in the Balmer lines than in the optical continuum.) However, due to the limited rest-frame period considered here, we consider this result tentative.

We do not see any strong FWHM or line profile variations in our sample of high-luminosity quasars. However, at the low resolution of our spectra (about $1200 \mathrm{~km} \mathrm{~s}^{-1}$ at $5000 \AA$ ), our ability to detect such variations is limited to dramatic changes only.

\subsection{Line-Continuum Cross-Correlations}

The ultimate objective of our program is to detect and measure a time delay between the continuum and the line flux variations in high-luminosity AGNs. The significant continuum and line variations that we have observed during a decade demonstrate that, at least in principle, such a measurement may be feasible. However, an actual estimate of a delay requires (1) high variability amplitude in both the line and the continuum light curves and (2) light curves with at least a few, reasonably sampled, largeamplitude "events," i.e., changes of sign in slope, that permit unambiguous matching between continuum and line variations. Examining the light curves of the six quasars with emission-line data at the current stage of our project, all but one currently suffer 
from either low-variability amplitude in the emission-line light curves or monotonically increasing or decreasing continuum light curves. In four objects the $\mathrm{C}$ IV light curve tracks the continuum's monotonic variation (SBS 1116+603, SBS 1233+594, SBS 1425+606, and HS 1700+6416). We note that the monotonic variation trend for these objects is unlikely to continue indefinitely. We expect that at some point, there will be a "break" in each of these cases, permitting the eventual measurement of a lag. However, the one current exception to this state of affairs is S5 0836+71; although the data for this quasar are still not ideal in terms of the criteria above, they do allow a preliminary measurement of the emission line to continuum lag. The continuum light curve for this object displays a general rise until 2002 June, followed by a sharp drop in flux. This light curve has the largest variation among all our monitored quasars, and the light curves of both $\mathrm{C}_{\mathrm{IV}}$ and $\mathrm{C}$ III] display the largest variations among all the emission-line light curves. Both emission-line light curves seem to follow the general trend of the continuum light curves, although the $C$ Iv light curve seems to have a much larger time lag than the $\mathrm{C}$ III] light curve.

To quantify the time lag, we use two methods for crosscorrelating the line and continuum light curves. The first method is the interpolated cross-correlation function (ICCF), as implemented by White \& Peterson (1994; see also the review by Gaskell 1994). The second method is the $z$-transformed discrete correlation function (ZDCF) of Alexander (1997). The two methods yield similar results for the current data, and we use only the ICCF results in the following analyses. The results of the crosscorrelation analysis are presented in Figure 5.

The CCFs for S5 $0836+71$ show enough structure to permit estimation of a time lag. The clearest case is the correlation between the $\mathrm{C}$ IV line and the continuum. To quantify the possible time lag and its uncertainty, we use the model-independent FR/RSS Monte Carlo method of Peterson et al. (1998b, 2005). In this method, each Monte Carlo simulation is composed of two parts. The first is a "random subset selection" (RSS) procedure that consists of randomly drawing, with replacement, from a light curve of $N$ points a new sample of $N$ points, while preserving the temporal order. The second part is "flux randomization" (FR), in which the observed fluxes are altered by random Gaussian deviates scaled to the uncertainty ascribed to each point. The two resampled and altered time series are cross-correlated using the ICCF method, and the centroid of the CCF is computed. We used $\sim 10,000$ Monte Carlo realizations to build up a cross-correlation centroid distribution (CCCD; e.g., Maoz \& Netzer 1989). The mean of the distribution is taken to be the time lag, and the uncertainty is determined as the range that contains $68 \%$ of the Monte Carlo realizations in the CCCD, and thus would correspond to $1 \sigma$ uncertainties for a normal distribution.

We find the time lag between the $\mathrm{C}$ IV line and the continuum of S5 $0836+71$ to be $595_{-110}^{+86}$ days, or $188_{-37}^{+27}$ days in the quasar rest frame. However, $17 \%$ of the CCCD simulations failed to produce significant centroid measurements. This may indicate that the quality of the data render this analysis premature. The cross-correlation of the $\mathrm{C}$ III] light curve with the continuum yields a peak around zero time lag. A formal CCCD gives $-152_{-182}^{+199}$ days, which is $-48_{-57}^{+63}$ days in the rest frame, but the CCCD function is not simple, with several peaks within this uncertainty range. We note that the only AGNs in which (rather uncertain) $\mathrm{C}$ III] time lags have been estimated to date are NGC 5548 (Peterson et al. 2004 and references therein) and NGC 4151 (Metzroth et al. 2006).

Until recently, only four AGNs had measured C IV reverberation time lags: NGC 3783, NGC 5548, NGC 7469, and 3C 390.3

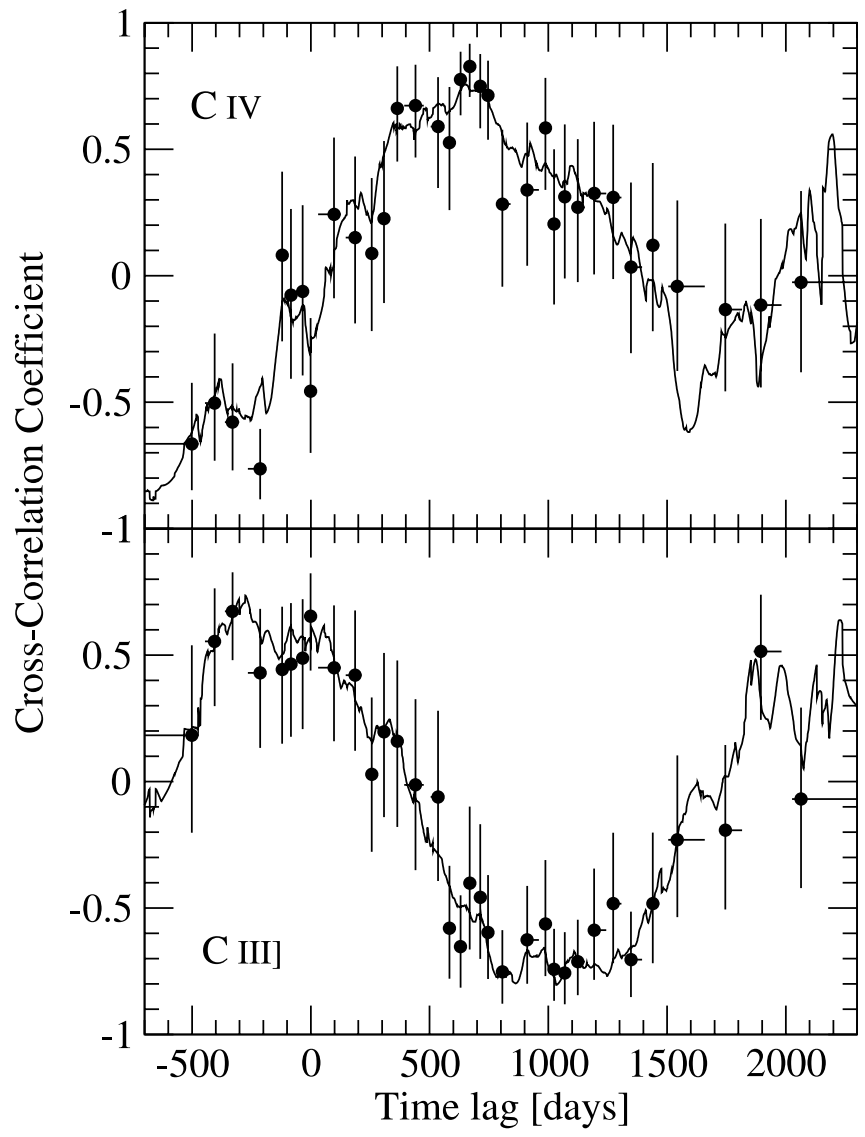

FIG. 5.-Cross-correlation functions, ICCF (solid curves) and ZDCF (circles with error bars), between the continuum and the emission-line (C IV, top; C III], bottom) light curves of S5 $0836+71$ from Fig. 3 .

(see Peterson et al. 2004 for a summary). Peterson et al. (2005) have now measured the $\mathrm{C}$ IV time lag also for the low-luminosity Seyfert galaxy NGC 4395, which is 4 orders of magnitude lower in luminosity than those four AGNs, permitting a first estimate of the BLR radius-luminosity relationship for the $\mathrm{C}$ IV-emitting region. Our preliminary determination of the $\mathrm{C}$ IV time lag for S5 $0836+71$ allows us to extend this relation to 7 orders of magnitude in luminosity. In Figure 6 we show the data as presented by Peterson et al. (2005), as well as their adopted best-fit relation (dotted line), to which we have added the data point for S5 $0836+71$. The rest-frame UV luminosity of S5 $0836+71$ has been computed from the mean flux during our observations. We have corrected the luminosity for a Galactic extinction of $A_{V}=0.101$ from Schlegel et al. (1998) using the extinction curve of Cardelli et al. (1989). We find the luminosity to be $\lambda L_{\lambda}(1350 \AA)=(1.12 \pm$ $0.16) \times 10^{47} \mathrm{ergs} \mathrm{s}^{-1}$. Our new data point deviates from the extrapolation of the fit by Peterson et al. (2005), which would predict a rest-frame time delay for S5 $0836+71$ of 372-1865 days. We note that, due to the current length of our program, we are sensitive to time lags of up to about 1000 days in the observer frame.

Most previous reverberation mapping experiments have measured the Balmer emission lines, in particular $\mathrm{H} \beta$. For the four AGNs above, $\mathrm{H} \beta$ reverberation measurements exist, in addition to the $C$ IV measurements (Peterson et al. 2004). The scaling between the BLR sizes of the two lines is important, since it is the means by which the BLR size from $\mathrm{C}$ IV reverberation mapping of high-luminosity quasars can be compared to the BLR size from $\mathrm{H} \beta$ reverberation mapping of Seyferts and low-luminosity quasars. For NGC 3783, NGC 5548, and NGC 7469 the $\mathrm{H} \beta$ 


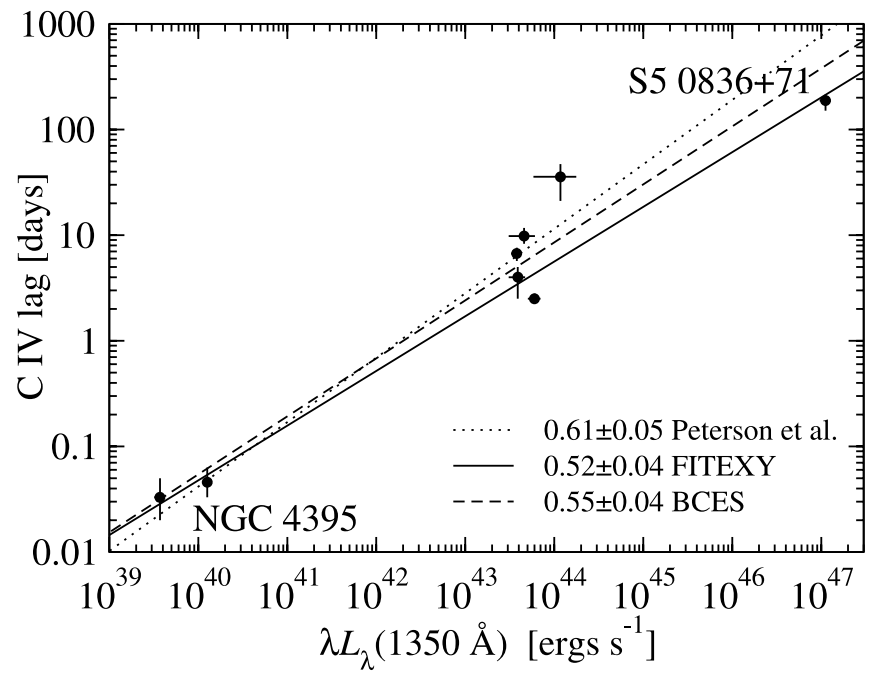

FIG. 6.- Size-luminosity relationship based on the $\mathrm{C}$ IV $\lambda 1549$ emission line and the UV continuum. Data (circles) are reproduced from Peterson et al. (2005), to which we add S5 $0836+71$ from the current study. The new data point deviates somewhat from the best-fit relation found by Peterson et al. (dotted line). Our own linear fits to the data are marked with a solid line for the FITEXY method and a dashed line for the BCES method. The power-law slopes of the three relations are indicated.

delays are factors of $2.55,1.98$, and 1.8 (which average to $\sim 2$ ) larger than the C IV delays, respectively, but for 3C 390.3 the $\mathrm{C}$ IV delay is larger than the $\mathrm{H} \beta$ delay by a factor of 1.5 . Thus, the relation between the $\mathrm{C}$ IV and $\mathrm{H} \beta$ BLR sizes is still unclear, due to the small number of objects with reverberation measurements in both lines, and the small spread in luminosity among these four AGNs. BLR stratification suggests that the C IV emission should arise interior to the $\mathrm{H} \beta$ emission. This is confirmed by the three Seyfert $1 \mathrm{~s}$ but not by the measurements of 3C 390.3. Also, the very small spread of these four AGNs in luminosity does not allow a meaningful scaling of the $\mathrm{H} \beta$ BLR size to the $\mathrm{C}$ IV BLR size; broadening the luminosity range is essential.

We have performed a linear regression for the C IV BLR sizeluminosity relation using all current data points in Figure 6. We have used the two methods that are described in detail in Kaspi et al. (2005): (1) the FITEXY from Press et al. (1992, p. 660), which is based on an iterative process to minimize $\chi^{2}$ and in which we adopted the Tremaine et al. (2002) procedure to account for the intrinsic scatter in the data, and (2) the bivariate correlated errors and intrinsic scatter (BCES) regression method of Akritas \& Bershady (1996). Both methods take into account the uncertainty in both coordinates and the intrinsic scatter around a straight line. The fit for the data we find from the FITEXY method (solid line in Fig. 6) is

$$
\frac{R_{\mathrm{BLR}}}{10 \text { lt-days }}=(0.17 \pm 0.04)\left[\frac{\lambda L_{\lambda}(1350 \AA)}{10^{43} \mathrm{ergs} \mathrm{s}^{-1}}\right]^{0.52 \pm 0.04},
$$

and for the BCES method (dashed line in Fig. 6) it is

$$
\frac{R_{\mathrm{BLR}}}{10 \text { lt-days }}=(0.24 \pm 0.06)\left[\frac{\lambda L_{\lambda}(1350 \AA)}{10^{43} \mathrm{ergs} \mathrm{s}^{-1}}\right]^{0.55 \pm 0.04} \text {. }
$$

The Pearson correlation coefficient is 0.97 with a significance level of $7 \times 10^{-5}$, and the Spearman rank-order correlation is 0.83 with a significance level of 0.01 . The intrinsic scatter we find using the Tremaine et al. (2002) procedure is 54\%. The slope we find for the relation between the C IV BLR size and UV luminosity is very close to the one found by Kaspi et al. (2005) between $\mathrm{H} \beta$ BLR size and the UV luminosity $(\sim 0.55)$.

The mean FWHM of the $\mathrm{C}$ IV line measured from the mean spectrum of S5 $0836+71$ is about $9700 \mathrm{~km} \mathrm{~s}^{-1}$. Using equation (5) of Kaspi et al. (2000) and the time lag of 188 days, we estimate the central mass of S5 $0836+71$ at about $2.6 \times 10^{9} M_{\odot}$. This is the highest mass directly measured for the black hole in an AGN using reverberation mapping. The quasar with the highest directly measured mass so far, 3C 273 (=PG 1226+023), has a mass of $8.9 \times 10^{8} M_{\odot}, \lambda L_{\lambda}(1350 \AA)=2.0 \times 10^{46} \mathrm{ergs} \mathrm{s}^{-1}$, and $\lambda L_{\lambda}(5100 \AA)=9.1 \times 10^{45} \mathrm{ergs} \mathrm{s}^{-1}$. Thus, S5 $0836+71$ has a factor of 3 higher mass and a factor $\sim 6$ higher luminosity than $3 \mathrm{C} 273$.

The central mass of S5 $0836+71$ corresponds to an Eddington luminosity of $L_{\mathrm{Edd}}=3.9 \times 10^{47} \mathrm{ergs} \mathrm{s}^{-1}$ (for solar abundance gas $L_{\text {Edd }} \approx 1.5 \times 10^{38} M / M_{\odot}$; e.g., Shaviv 1998). Using $\lambda L_{\lambda}(5100 \AA)=$ $6.4 \times 10^{46} \mathrm{ergs} \mathrm{s}^{-1}$ for S5 $0836+71$ and a bolometric correction of 5.6 from Marconi et al. (2004; eq. [21]) we find a bolometric luminosity, $L_{\mathrm{bol}}=3.6 \times 10^{47} \mathrm{ergs} \mathrm{s}^{-1}$. Thus, this object is accreting close to its Eddington luminosity, with $L_{\mathrm{bol}} / L_{\mathrm{Edd}} \approx 0.9$. We note that using equation (7) from Vestergaard \& Peterson (2006) to estimate the black hole mass from the UV luminosity and the $\mathrm{C}$ IV FWHM, one obtains $M_{\mathrm{BH}}=1.8 \times 10^{10} M_{\odot}$, which is 7 times higher than our tentative measurement from reverberation mapping.

The high Eddington ratio that we obtain is similar to that found for many other quasars (e.g., Shemmer et al. 2004; Baskin \& Laor 2005). However, we note that there are several caveats that could influence our result. The first is the tentative nature of our measurement of the time lag for the $\mathrm{C}$ IV line. One should keep in mind the possibility that the correlation and delay that we have measured in S5 0836+71 might be a chance coincidence, since it is the single object in which we have found a correlation, out of six objects in which we have searched for one. Another concern is our measured luminosity of the object. Since S5 $0836+71$ is the radio loudest object in our spectrophotometric sample, it could be that a jet-linked, beamed optical continuum is influencing the variability and the continuum-flux measurement. While a beamed component might influence the variability on small timescales (the continuum light curve does show variability on timescales of months) the $\mathrm{C}$ IV line light curve is smooth, and its response is to the long-timescale variations seen in the continuum light curve. We have also checked the C Iv EWs of the six quasars monitored at the HET, of which three are radio loud and three are radio quiet. For the radio-loud quasars, the observed EWs are 33, 68, and $101 \AA$, while for the radio-quiet ones the EWs are 57, 68, and $90 \AA$. Thus, both classes have comparable C Iv EWs, and there is no clear difference that might indicate that there is an additional contribution to the UV continuum luminosity of the radio-loud quasars.

\section{SUMMARY}

We have presented first results from our long-term program to monitor high-luminosity, high-redshift quasars, with the objective of eventually measuring the sizes of their broad-line regions and the masses of their central black holes. All 11 quasars in our photometrically monitored sample show continuum variability of $10 \%-70 \%$ over a rest-frame period of $2-3 \mathrm{yr}$. Compared to previous studies of lower luminosity AGNs, the continuum variability amplitude of higher luminosity quasars over the same rest-frame interval appears to be smaller in the current highluminosity sample. Six of the 11 quasars have been monitored spectroscopically over the past $\sim 5 \mathrm{yr}(1.2-1.5 \mathrm{yr}$ in the quasar rest frames) using the HET. None of the quasars show Ly $\alpha$ 
variations, at a level of $<7 \%$, and this nonvariability may be a generic feature of high-luminosity AGNs. However, we do detect variable $\mathrm{C}$ III] and $\mathrm{C}$ IV broad-line fluxes, whenever these lines are in our observed spectral range. In several cases, these lines track the continuum variations in the same quasar. The variations in the broad $\mathrm{C}_{\mathrm{III}}$ ] and $\mathrm{C}$ IV lines are higher than Balmer line variations in low-luminosity quasars over the same rest-frame interval, but this result is tentative, in view of the still-limited spectroscopic monitoring period.

In one object, $\mathrm{S} 50836+71$, we tentatively measure the $\mathrm{C}$ IV restframe time lag behind the continuum emission to be $188_{-37}^{+27}$ days. This is lower than expected from an extrapolation of the $\mathrm{C}$ IV BLR size-luminosity relation measured at lower luminosities. Our measurement permits tracing this relation over 7 orders of magnitude in AGN luminosity. From this time lag we estimate a black hole mass of $2.6 \times 10^{9} M_{\odot}$ in S5 $0836+71$, the largest ever measured by this technique.

Our results to date demonstrate that reverberation mapping of high-redshift, high-luminosity quasars may be feasible. Based on our past experience with quasar reverberation (Maoz et al. 1994; Kaspi et al. 1996a, 2000), we hope that with $\approx 5$ more years of similar data we will be able to establish a reliable BLR size for S5 0836+71, and perhaps for several other objects in our sample. The unpredictability of quasar light curves, together with our lack of knowledge about the response amplitudes and timescales of the UV lines, makes it difficult to estimate our chances of success. If we do succeed, the combined reverberation results from this program and those from a program being carried out on very low-luminosity AGNs will cover the entire AGN luminosity range and much of cosmic time, including the peak era of black hole growth for the most massive black holes and galaxies. This will enable a direct measurement of the black hole mass in these AGNs and a more reliable indirect mass measurement for all AGNs.
We are grateful to the staffs of the WO and HET for their great help in carrying out this long-term program. Special thanks go to John Dann, Ezra Mashal, and Sami Ben-Gigi of the WO, and to Gary Hill of the HET for devoted technical support of this project through the years. We thank Ari Laor for helpful discussions, and Mike Eracleous and Larry Ramsey for their support. We thank the anonymous referee for several valuable suggestions. The Hobby-Eberly Telescope (HET) is a joint project of the University of Texas at Austin, Pennsylvania State University, Stanford University, Ludwig-Maximillians-Universität München, and GeorgAugust-Universität Göttingen. The HET is named in honor of its principal benefactors, William P. Hobby and Robert E. Eberly. The Marcario Low-Resolution Spectrograph is named for Mike Marcario of High Lonesome Optics, who fabricated several optics for the instrument but died before its completion; it is a joint project of the HET partnership and the Instituto de Astronomía de la Universidad Nacional Autónoma de México. We gratefully acknowledge the financial support of the Colton Foundation at Tel Aviv University (S. K.), the Zeff Fellowship at the Technion (S. K.), NASA LTSA grant NAG5-13035 (W. N. B., D. P. S., and O. S.), the Israel Science Foundation grant 232/03 (H. N.), and NSF grant AST 03-07582 (D. P. S.). This research has made use of the NASA/IPAC Extragalactic Database (NED), which is operated by the Jet Propulsion Laboratory, California Institute of Technology, under contract with the National Aeronautics and Space Administration. Funding for the creation and distribution of the SDSS and SDSS-II has been provided by the Alfred P. Sloan Foundation, the Participating Institutions, the National Science Foundation, the US Department of Energy, the National Aeronautics and Space Administration, the Japanese Monbukagakusho, the Max Planck Society, and the Higher Education Funding Council for England. The SDSS Web site is http://www.sdss.org/.
Abazajian, K., et al. 2005, AJ, 129, 1755

Akritas, M. G., \& Bershady, M. A. 1996, ApJ, 470, 706

Alexander, T. 1997, in Astronomical Time Series, ed. D. Maoz, A. Sternberg, \&

E. M. Leibowitz (Dordrecht: Kluwer), 163

Baldwin, J. A. 1977, ApJ, 214, 679

Barger, A. J., Cowie, L. L., Mushotzky, R. F., Yang, Y., Wang, W.-H., Steffen, A. T., \& Capak, P. 2005, AJ, 129, 578

Baskin, A., \& Laor, A. 2005, MNRAS, 356, 1029

Bentz, M. C., Peterson, B. M., Pogge, R. W., Vestergaard, M., \& Onken, C. A. 2006, ApJ, 644, 133

Cardelli, J. A., Clayton, G. C., \& Mathis, J. S. 1989, ApJ, 345, 245

Cid Fernandes, R., Sodré, L., Jr., \& Vieira da Silva, L., Jr. 2000, ApJ, 544, 123

Clavel, J., et al. 1991, ApJ, 366, 64

Collier, S. J., et al. 1998, ApJ, 500, 162

Edelson, R., Turner, T. J., Pounds, K., Vaughan, S., Markowitz, A., Marshall, H., Dobbie, P., \& Warwick, R. 2002, ApJ, 568, 610

Gaskell, C. M. 1994, in ASP Conf. Ser. 69, Reverberation Mapping of the BroadLine Region in AGNs, ed. P. Gondhalekar, K. Horne, \& B. M. Peterson (San Francisco: ASP), 111

Giveon, U., Maoz, D., Kaspi, S., Netzer, H., \& Smith, P. S. 1999, MNRAS, 306,637

Hill, G. J., Nicklas, H. E., MacQueen, P. J., Tejada, C., Cobos Duenas, F. J., \& Mitsch, W. 1998, Proc. SPIE, 3355, 375

Jester, S., et al. 2005, AJ, 130, 873

Jiang, L., Fan, X., Ivezic, Z., Richards, G. T., Schneider, D. P., Strauss, M. A., \& Kelly, B. C. 2007, ApJ, 656, 680

Kaspi, S., Ibbetson, P. A., Mashal, E., \& Brosch, N. 1995, Wise Obs. Tech. Rep. 6

Kaspi, S., Maoz, D., Netzer, H., Peterson, B. M., Vestergaard, M., \& Jannuzi, B. T. 2005, ApJ, 629, 61

Kaspi, S., Smith, P. S., Maoz, D., Netzer, H., \& Jannuzi, B. T. 1996a, ApJ, 471, L75

Kaspi, S., Smith, P. S., Netzer, H., Maoz, D., Jannuzi, B. T., \& Giveon, U. 2000, ApJ, 533, 631

Kaspi, S., et al. 1996b, ApJ, 470, 336
Kollmeier, J. A., et al. 2006, ApJ, 648, 128

Korista, K. T., et al. 1995, ApJS, 97, 285

Laor, A., \& Brandt, W. N. 2002, ApJ, 569, 641

Lawrence, A., \& Papadakis, I. 1993, ApJ, 414, L85

Maoz, D., Markowitz, A., Edelson, R., \& Nandra, K. 2002, AJ, 124, 1988

Maoz, D., \& Netzer, H. 1989, MNRAS, 236, 21

Maoz, D., Smith, P. S., Jannuzi, B. T., Kaspi, S., \& Netzer, H. 1994, ApJ, 421, 34

Maoz, D., et al. 1993, ApJ, 409, 28

Marconi, A., Risaliti, G., Gilli, R., Hunt, L. K., Maiolino, R., \& Salvati, M. 2004, MNRAS, 351, 169

Markowitz, A., et al. 2003, ApJ, 593, 96

McLure, R. J., \& Dunlop, J. S. 2004, MNRAS, 352, 1390

McLure, R. J., \& Jarvis, M. J. 2002, MNRAS, 337, 109

Metzroth, K. G., Onken, C. A., \& Peterson, B. M. 2006, ApJ, 647, 901

Morgan, N. D., Gregg, M. D., Wisotzki, L., Becker, R., Maza, J., Schechter, P. L., \& White, R. L. 2003, AJ, 126, 696

Netzer, H. 2003, ApJ, 583, L5

Netzer, H., \& Peterson, B. M. 1997, in Astronomical Time Series, ed. D. Maoz,

A. Sternberg, \& E. Leibowitz (Dordrecht: Kluwer), 85

Netzer, H., et al. 1996, MNRAS, 279, 429

O'Brien, P. T., et al. 1998, ApJ, 509, 163

O’Neill, P. M., Nandra, K., Papadakis, I. E., \& Turner, T. J. 2005, MNRAS, 358,1405

Peterson, B. M. 1993, PASP, 105, 247

. 2006, in Physics of Active Galactic Nuclei at All Scales, ed. D. Alloin, R. Johnson, \& P. Lira (Berlin: Springer), 77

Peterson, B. M., Wanders, I., Bertram, R., Hunley, J. F., Pogge, R. W., \& Wagner, R. M. 1998a, ApJ, 501, 82

Peterson, B. M., Wanders, I., Horne, K., Collier, S., Alexander, T., Kaspi, S., \& Maoz, D. 1998b, PASP, 110, 660

Peterson, B. M., et al. 2004, ApJ, 613, 682 2005, ApJ, 632, 799 (erratum 641, 638 [2006])

Press, W. H., Teukolsky, S. A., Vetterling, W. T., \& Flannery, B. P. 1992, Numerical Recipes in FORTRAN (2nd ed.; Cambridge: Cambridge Univ. Press) 
Ramsey, L. W., et al. 1998, Proc. SPIE, 3352, 34

Reichert, G. A., et al. 1994, ApJ, 425, 582

Reimers, D., Hagen, H.-J., Schramm, J., Kriss, G. A., \& Shull, J. M. 2005, A\&A, 436, 465

Riess, A. G., et al. 2004, ApJ, 607, 665

Rodriguez-Pascual, P. M., et al. 1997, ApJS, 110, 9

Schlegel, D. J., Finkbeiner, D. P., \& Davis, M. 1998, ApJ, 500, 525

Schmidt, M., \& Green, R. F. 1983, ApJ, 269, 352

Schneider, D. P., et al. 2005, AJ, 130, 367

Shaviv, N. J. 1998, ApJ, 494, L193

Shemmer, O., Netzer, H., Maiolino, R., Oliva, E., Croom, S., Corbett, E., \& di Fabrizio, L. 2004, ApJ, 614, 547

Shemmer, O., Uttley, P., Netzer, H., \& McHardy, I. M. 2003, MNRAS, 343, 1341

Spergel, D. N., et al. 2003, ApJS, 148, 175

Steffen, A. T., Strateva, I., Brandt, W. N., Alexander, D. M., Koekemoer, A. M., Lehmer, B. D., Schneider, D. P., \& Vignali, C. 2006, AJ, 131, 2826

Strateva, I. V., Brandt, W. N., Schneider, D. P., Vanden Berk, D. G., \& Vignali, C. 2005, AJ, 130, 387

Tremaine, S., et al. 2002, ApJ, 574, 740
Trevese, D., Stirpe, G., Vagnetti, F., Zitelli, V., \& Paris, D. 2006, in ASP Conf. Ser. 360, AGN Variability from X-Rays to Radio Waves, ed. C. M. Gaskell et al. (San Francisco: ASP), (astro-ph/0408075)

Ulrich, M.-H., Courvoisier, T. J.-L., \& Wamsteker, W. 1993, ApJ, 411, 125

Uttley, P., McHardy, I. M., \& Papadakis, I. E. 2002, MNRAS, 332, 231

Vanden Berk, D. E., et al. 2004, ApJ, 601, 692

Véron-Cetty, M.-P., \& Véron, P. 1993, ESO Scientific Rep. 13, A Catalogue of Quasars and Active Nuclei (6th ed.; Garching: ESO)

Vestergaard, M. 2002, ApJ, 571, 733

Vestergaard, M., \& Peterson, B. M. 2006, ApJ, 641, 689

Wanders, I., et al. 1997, ApJS, 113, 69

Welsh, W., et al. 2000, BAAS, 32, 1458

White, R. J., \& Peterson, B. M. 1994, PASP, 106, 879

Wills, B. J., Netzer, H., Brotherton, M. S., Han, M., Wills, D., Baldwin, J. A., Ferland, G. J., \& Browne, I. W. A. 1993, ApJ, 410, 534

Woo, J.-H., \& Urry, C. M. 2002, ApJ, 581, L5

Wu, X.-B., Wang, R., Kong, M. Z., Liu, F. K., \& Han, J. L. 2004, A\&A, 424, 793

York, D. G., et al. 2000, AJ, 120, 1579

Yu, Q., Lu, Y., \& Kauffmann, G. 2005, ApJ, 634, 901 